\title{
Stable rationality of quadric surface bundles over surfaces
}

\author{
by \\ BRENDAN HASSETT \\ Brown University \\ Providence, RI, U.S.A. \\ Alena Pirutka \\ New York University \\ New York, NY, U.S.A. \\ YURI TSCHINKEL \\ New York University \\ New York, NY, U.S.A.
}

\section{Introduction}

Is a deformation of a smooth rational (or irrational) projective variety still rational (or irrational)? The main goal of this paper is to show that rationality is not deformationinvariant for families of smooth complex projective varieties of dimension 4. Examples along these lines are known when singular fibers are allowed, e.g., smooth cubic threefolds (which are irrational) may specialize to cubic threefolds with ordinary double points (which are rational), while smooth cubic surfaces (which are rational) may specialize to cones over elliptic curves. Totaro shows that specializations of rational varieties need not be rational in higher dimensions if mild singularities are allowed [T2]. However, de Fernex and Fusi [dFF] show that the locus of rational fibers in a smooth family of projective complex threefolds is a countable union of closed subsets on the base.

Let $S$ be a smooth projective rational surface over the complex numbers with function field $K=\mathbb{C}(S)$. A quadric surface bundle consists of a fourfold $X$ and a flat projective morphism $\pi: X \rightarrow S$ such that the generic fiber $Q / K$ of $\pi$ is a smooth quadric surface. We assume that $\pi$ factors through the projectivization of a rank-4 vector bundle on $S$ such that the fibers are (possibly singular) quadric surfaces; see $\S 3$ for relevant background.

TheOREM 1. There exist smooth families of complex projective fourfolds $\phi: \mathcal{X} \rightarrow B$ over connected varieties $B$, such that for every $b \in B$ the fiber $\mathcal{X}_{b}=\phi^{-1}(b)$ is a quadric surface bundle over $\mathbb{P}^{2}$, and satisfying the following properties:

(1) for very general $b \in B$ the fiber $\mathcal{X}_{b}$ is not stably rational; 
(2) the set of points $b \in B$ such that $\mathcal{X}_{b}$ is rational is dense in $B$ for the Euclidean topology.

Concretely, we consider smooth hypersurfaces

$$
X \subset \mathbb{P}^{2} \times \mathbb{P}^{3}
$$

of bidegree $(2,2)$; projection onto the first factor gives the quadric surface bundle.

Our approach has two key elements. First, we apply the technique of the decomposition of the diagonal [V2], [CTPa], [CTPo], [T1] to show that very general $X \subset \mathbb{P}^{2} \times \mathbb{P}^{3}$ of bidegree $(2,2)$ fail to be stably rational. The point is to identify a degenerate quadric surface fibration, with non-trivial second unramified cohomology and mild singularities. The analogous degenerate conic bundles over $\mathbb{P}^{2}$ are the Artin-Mumford examples; deforming these allows one to show that very general conic bundles over $\mathbb{P}^{2}$ with large degeneracy divisor fail to be stably rational [HKT]. Second, quadric surface bundles are rational over the base whenever they admit a section, indeed, whenever they admit a multisection of odd degree. If the base is rational, then the total space is rational as well; this can be achieved over a dense set of the moduli space [H], [V3]. This technique also yields rationality for a dense family of cubic fourfolds containing a plane; no cubic fourfolds have been shown not to be stably rational.

Theorem 1 is proven in $\S 7$, which may serve as roadmap for the steps of our argument.

This paper is inspired by the approach of Voisin [V3], who also considers fourfolds birational to quadric surface bundles. While our proof of rationality is similar, the analysis of unramified cohomology relies on work of Pirutka $[\mathrm{P}]$ and Colliot-Thélène and Ojanguren [CTO].

\section{Acknowledgments}

The first author was partially supported through NSF grant 1551514 . We are grateful to Jean-Louis Colliot-Thélène and Burt Totaro for helpful comments on drafts of this manuscript. Our paper benefited greatly from the careful reading by the referees.

\section{The specialization method}

We recall implications of the "integral decomposition of the diagonal and specialization" method, following [V2], [CTPa], and [P].

A projective variety $X$ over a field $k$ is universally $\mathrm{CH}_{0}$-trivial if, for all field extensions $k^{\prime} / k$, the natural degree homomorphism from the Chow group of zero-cycles

$$
\mathrm{CH}_{0}\left(X_{k^{\prime}}\right) \longrightarrow \mathbb{Z}
$$


is an isomorphism. Examples include smooth $k$-rational varieties. More complicated examples arise as follows.

Example 2. [CTPo, Lemmas 2.3 and 2.4] Let $X=\bigcup_{i} X_{i}$ be a projective, reduced, geometrically connected variety over a field $k$ such that the following conditions hold:

- each irreducible component $X_{i}$ is geometrically irreducible and $k$-rational, with isolated singularities;

- each intersection $X_{i} \cap X_{j}$ is either empty or has a zero-cycle of degree 1 .

Then, $X$ is universally $\mathrm{CH}_{0}$-trivial.

A projective morphism

$$
\beta: \widetilde{X} \longrightarrow X
$$

of $k$-varieties is universally $\mathrm{CH}_{0}$-trivial if for all extensions $k^{\prime} / k$ the push-forward homomorphism

$$
\beta_{*}: \mathrm{CH}_{0}\left(\widetilde{X}_{k^{\prime}}\right) \longrightarrow \mathrm{CH}_{0}\left(X_{k^{\prime}}\right)
$$

is an isomorphism.

Proposition 3. ([CTPa, Proposition 1.8]) Let

$$
\beta: \widetilde{X} \longrightarrow X
$$

be a projective morphism such that for every schematic point $x$ of $X$, the fiber $\beta^{-1}(x)$, considered as a variety over the residue field $\varkappa(x)$, is universally $\mathrm{CH}_{0}$-trivial. Then, $\beta$ is universally $\mathrm{CH}_{0}$-trivial.

For example, if $X$ is a smooth projective variety and

$$
\beta: \mathrm{Bl}_{Z}(X) \longrightarrow X
$$

is a blow up of a smooth subvariety $Z \subset X$, then $\beta$ is a universally $\mathrm{CH}_{0}$-trivial morphism, since all fibers over (schematic) points are projective spaces. More interesting examples arise as resolutions of singularities of certain singular projective varieties.

Examples of failure of universal $\mathrm{CH}_{0}$-triviality are given by smooth projective varieties $X$ with non-trivial Brauer group $\operatorname{Br}(X)$, or more generally, by varieties with nontrivial higher unramified cohomology $[\mathrm{CTPa}, \S 1]$. The following specialization argument is the key to recent advances in investigations of stable rationality.

Theorem 4. ([V2, Theorem 2.1] and [CTPa, Theorem 2.3]) Let

$$
\phi: \mathcal{X} \longrightarrow B
$$


be a flat projective morphism of complex varieties with smooth generic fiber. Assume that there exists a point $b \in B$ such that the fiber

$$
X:=\phi^{-1}(b)
$$

satisfies the following conditions:

- the group $H_{n r}^{2}(\mathbb{C}(X) / \mathbb{C}, \mathbb{Z} / 2 \mathbb{Z})$ is non-trivial;

- $X$ admits a desingularization

$$
\beta: \widetilde{X} \longrightarrow X
$$

such that the morphism $\beta$ is universally $\mathrm{CH}_{0}$-trivial.

Then, a very general fiber of $\phi$ is not stably rational.

\section{Quadric surface bundles}

Let $S$ be a smooth projective variety over $\mathbb{C}$. Suppose that $\pi: X \rightarrow S$ is a quadric surface bundle, i.e., a flat projective morphism from a variety such that the generic fiber $Q$ is a smooth quadric surface. We assume it admits a factorization

$$
X \hookrightarrow \mathbb{P}(V) \longrightarrow S,
$$

where $V \rightarrow S$ is a rank- 4 vector bundle and the fibers of $\pi$ are expressed as quadric surfaces in the fibers of $\mathbb{P}(V) \rightarrow S$. There is a well-defined degeneracy divisor $D \subset S$ corresponding to where the associated quadratic form drops rank.

Trivializing $V$ over an open cover of $S, X$ may be expressed using a symmetric $4 \times 4$ matrix $\left(a_{i j}\right)$ :

$$
\sum_{i, j=1}^{4} a_{i j} x_{i} x_{j}=0 .
$$

The local equation for $D$ is the determinant $\operatorname{det}\left(a_{i j}\right)$. Note that $D$ has multiplicity $\geqslant 2$ where the rank of fibers is less than 3 . Indeed, the hypersurface

$$
\left\{\operatorname{det}\left(a_{i j}\right)=0\right\} \subset \mathbb{P}_{\left(a_{i j}\right)}^{9}
$$

is singular precisely where all the $3 \times 3$ minors vanish.

\subsection{Rationality of quadric bundles}

It is well known that $Q$ is rational over $K=\mathbb{C}(S)$ if and only if $Q(K) \neq \varnothing$, i.e., when $\pi$ admits a rational section. A theorem by Springer [S] implies that $Q(K) \neq \varnothing$ provided $Q\left(K^{\prime}\right) \neq \varnothing$ for some extension $K^{\prime} / K$ of odd degree, i.e., when $\pi$ admits a rational multisection of odd degree. Thus, we obtain the following result. 
Proposition 5. Let $\pi: X \rightarrow S$ be a quadric surface bundle as above, with $S$ rational. Then, $X$ is rational provided $\pi$ admits a multisection of odd degree.

Our next step is to recast this in Hodge-theoretic terms.

Proposition 6. Let $\pi: X \rightarrow S$ be a quadric surface bundle as above, with $X$ smooth and $S$ rational. Then, $X$ is rational if it admits an integral $(2,2)$-class meeting the fibers of $\pi$ in odd degree.

Remark 7. See [CTV, Corollary 8.2] for results on the integral Hodge conjecture for quadric bundles over surfaces; these suffice for our application to quadric surface bundles over $\mathbb{P}^{2}$.

Proof. Let $F_{1}(X / S) \rightarrow S$ denote the relative variety of lines of $\pi$. Let $S_{\circ} \subset S$ denote the largest open subset such that $S_{\circ} \cap D$ is smooth and $X_{\circ}=X \times{ }_{S} S_{\circ}$. Then $F_{1}\left(X_{\circ} / S_{\circ}\right) \rightarrow$ $S$ 。 factors

$$
F_{1}\left(X_{\circ} / S_{\circ}\right) \stackrel{p}{\longrightarrow} T_{\circ} \longrightarrow S_{\circ}
$$

where the second morphism is a double cover branched along $S_{\circ} \cap D$ and the first morphism is an étale $\mathbb{P}^{1}$-bundle. In particular $F_{1}\left(X_{\circ} / S_{\circ}\right)$ is non-singular. Let $\alpha \in \operatorname{Br}\left(T_{\circ}\right)[2]$ denote the Brauer class arising from $p$.

Let $F$ be a resolution of the closure of $F_{1}\left(X_{\circ} / S_{\circ}\right)$ in $F_{1}(X / S)$ obtained by blowing up over the complement of $S_{\circ}$. The incidence correspondence between $X$ and $F_{1}(X / S)$,

$$
\Gamma^{\prime} \subset X \times{ }_{S} F_{1}(X / S)
$$

induces a correspondence $\Gamma$ between $X$ and $F$ and a homomorphism

$$
\Gamma_{*}: \mathrm{CH}^{2}(X) \longrightarrow \operatorname{Pic}(F)
$$

Let $\eta$ denote the generic point of $S$; there is a quadratic map

$$
\Xi: \operatorname{Pic}\left(F_{\eta}\right) \longrightarrow \mathrm{CH}^{2}\left(X_{\eta}\right)
$$

given by

$$
\Xi\left(\sum_{i} a_{i} x_{i}\right)=\frac{1}{2}\left(\sum_{i} a_{i} \ell\left(x_{i}\right)\right)^{2},
$$

where $\ell\left(x_{i}\right) \subset X_{\eta}$ is the line which corresponds to the point $x_{i} \in F_{\eta}$. In geometric terms, consider $Z \subset F_{\eta}$ a finite reduced subscheme with support on each component of $F_{\eta}$, e.g., a choice of $n$ lines from each ruling. Take the union of the corresponding rulings in $X_{\eta}$ and set $\Xi(Z) \subset X_{\eta}$ to be the $n^{2}$ points where the rulings cross. This is compatible with 
rational equivalence and yields the desired mapping. Thus, a divisor with odd degree on each geometric component of $F_{\eta}$ gives rise to a rational multisection of odd degree.

The correspondence $\Gamma$ and the mapping $\Xi$ guarantee that the following conditions are equivalent:

- $\alpha=0$;

- $F$ admits a divisor intersecting the generic fiber $F_{\eta}$ with odd degree on each component;

- $X$ admits a rational multisection of odd degree.

As the correspondence $\Gamma$ also acts at the level of Hodge classes we obtain the following fact: If $X$ admits an integral $(2,2)$-class intersecting the fibers of $\pi$ with odd degree, then $F$ admits an integral $(1,1)$-class intersecting the generic fiber $F_{\eta}$ with odd degree on each component.

Applying the Lefschetz $(1,1)$ theorem to $F$ and Proposition 5, we are done.

\subsection{A key example}

The generic fiber of $\pi$ is a quadric surface, hence admits a diagonal form

$$
Q=\langle 1, a, b, a b d\rangle
$$

i.e., is given by the equation

$$
s^{2}+a t^{2}+b u^{2}+a b d v^{2}=0
$$

where $a, b, d \in K^{\times}$and $(s, t, u, v)$ are homogeneous coordinates in $\mathbb{P}^{3}$. Note that, since $k:=\mathbb{C} \subset K$, this form is equivalent to the form $\langle 1,-a,-b, a b d\rangle$.

Theorem 3.17 in $[\mathrm{P}]$ gives a general formula for the unramified $H^{2}$ of the field $K(Q)$, in terms of the divisor of rational functions $a, b, d \in K^{\times}$, under the assumption that $d$ is not a square.

In $\S 4$ we will analyze the following special case.

Example 8. Consider the fourfold $X \subset \mathbb{P}^{2} \times \mathbb{P}^{3}$ given by

$$
y z s^{2}+x z t^{2}+x y u^{2}+F(x, y, z) v^{2}=0,
$$

where

$$
F(x, y, z)=x^{2}+y^{2}+z^{2}-2(x y+x z+y z) .
$$

Dehomogenize by setting $z=1$ to obtain a quadric surface over $k\left(\mathbb{P}^{2}\right)$ :

$$
y s^{2}+x t^{2}+x y u^{2}+F(x, y, 1) v^{2}=0 .
$$


Multiplying through by $x y$ and absorbing squares into the variables yields

$$
x S^{2}+y T^{2}+U^{2}+x y F(x, y, 1) V^{2}=0,
$$

which is of the form (3.1).

We compute the divisor $D \subset \mathbb{P}^{2}$ parameterizing singular fibers of $\pi: X \rightarrow \mathbb{P}^{2}$. This is reducible, consisting of the coordinate lines (with multiplicty 2 ) and a conic tangent to each of the lines:

$$
D=\left\{x^{2} y^{2} z^{2}\left(x^{2}+y^{2}+z^{2}-2(x y+x z+y z)\right)=0\right\} .
$$

Remark 9. Hypersurfaces of bidegree $(2,2)$ in $\mathbb{P}^{2} \times \mathbb{P}^{3}$ may also be regarded as conic bundles over the second factor. The degeneracy surface in $\mathbb{P}^{3}$ has degree 6 and at least eight nodes, corresponding to rank-1 fibers. As a byproduct of the proof of Theorem 1 , we obtain failure of stable rationality for very general conic bundles of this type.

\section{The Brauer group of the special fiber}

We refer the reader to $[\mathrm{CTO}, \S 1]$ and $[\mathrm{CT}]$ for basic properties of unramified cohomology.

Let $K$ be a field. We write

$$
H^{n}(K)=H^{n}(K, \mathbb{Z} / 2 \mathbb{Z})
$$

for its $n$th Galois cohomology with constant coefficients $\mathbb{Z} / 2 \mathbb{Z}$. Let $K=k(X)$ be the function field of an algebraic variety $X$ over $k=\mathbb{C}$, and let $\nu$ be a rank-1 discrete valuation of $K$, trivial on $k$. For $n \geqslant 1$, we have a natural homomorphism

$$
\partial_{\nu}^{n}: H^{n}(K) \longrightarrow H^{n-1}(\varkappa(\nu))
$$

where $\varkappa(\nu)$ is the residue field of $\nu$. The group

$$
H_{n r}^{n}(K / k):=\bigcap_{\nu} \operatorname{Ker}\left(\partial_{\nu}^{n}\right)
$$

is called the $n$th unramified cohomology of $K$. It is a stable birational invariant [CTO, Proposition 1.2] and vanishes if $X$ is stably rational [CTO, Corollary 1.2.1]. Recall that, for a smooth projective $X$, we have

$$
\operatorname{Br}(X)[2]=H_{n r}^{2}(k(X) / k) .
$$

The following proposition is similar to the examples in $[\mathrm{P}, \S 3.5]$. 
Proposition 10. Let $K=k(x, y)=k\left(\mathbb{P}^{2}\right), X \rightarrow \mathbb{P}^{2}$ be the quadric surface bundle defined in Example 8,

$$
\alpha=(x, y) \in \operatorname{Br}(K)[2],
$$

and $\alpha^{\prime}$ be its image in $H^{2}(k(X))$. Then, $\alpha^{\prime}$ is contained in $H_{n r}^{2}(k(X) / k)$ and is nontrivial. In particular,

$$
H_{n r}^{2}(k(X) / k) \neq 0 \text {. }
$$

Proof. Let $Q$ be the generic fiber of the natural projection $\pi: X \rightarrow \mathbb{P}^{2}$. Since the discriminant of $Q$ is not a square, the homomorphism

$$
H^{2}(K) \longrightarrow H^{2}(K(Q))
$$

is injective [A, p. 469], [K, Proposition 6.4.13]. Note that $\alpha \neq 0$, as the conic

$$
x S^{2}+y T^{2}=U^{2}
$$

has no rational points over $k(x, y)$; it follows that $\alpha^{\prime}$ is also non-trivial. It remains to show that for every rank-1 discrete valuation $\nu$ on $K(Q)$ that is trivial on $k$, we have $\partial_{\nu}\left(\alpha^{\prime}\right)=0$. (For simplicity, we write $\partial_{\nu}$ for $\partial_{\nu}^{2}$.) We use standard coordinates $x$ and $y$ (resp. $y$ and $z$, or $x$ and $z$ ) for the open charts of the projective plane. Let us first investigate the ramification of $\alpha$ on $\mathbb{P}^{2}$; from the definition, we only have the following non-trivial residues:

- $\partial_{x}(\alpha)=y$ at the line $L_{x}: x=0$, where we write $y$ for its class in the residue field $k(y)$ modulo squares;

- $\partial_{y}(\alpha)=x$ at the line $L_{y}: y=0$;

- $\partial_{z}(\alpha)=\partial_{z}(z, z y)=y$ at the line $L_{z}: z=0$, in coordinates $y$ and $z$ on $\mathbb{P}^{2}$.

Let $\mathfrak{o}_{\nu}$ be the valuation ring of $\nu$ in $K(Q)$ and consider the center of $\nu$ in $\mathbb{P}^{2}$. If $\mathfrak{o}_{\nu} \supset K$, then the $\partial_{\nu}\left(\alpha^{\prime}\right)=0$; hence, there are two cases to consider:

- the center is the generic point of a curve $C_{\nu} \subset \mathbb{P}^{2}$; we denote the corresponding residue map $\partial_{C_{\nu}}: H^{2}(K) \rightarrow H^{1}\left(\varkappa\left(C_{\nu}\right)\right)$;

- the center is a closed point $\mathfrak{p}_{\nu} \in \mathbb{P}^{2}$.

Codimension 1. The inclusion of discrete valuation rings $\mathfrak{o}_{\mathbb{P}^{2}, C_{\nu}} \subset \mathfrak{o}_{\nu}$ induces a commutative diagram [CTO, p. 143]

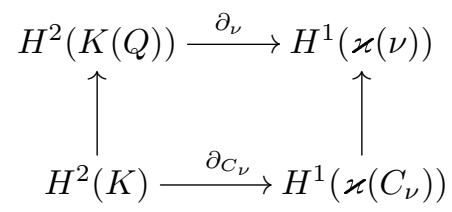


The right vertical arrow need not be the functorial homomorphism induced by inclusion of the residue fields when there is ramification.

Hence, we have the following cases:

(1) $C_{\nu}$ is different from $L_{x}, L_{y}$, and $L_{z}$. Then, $\partial_{C_{\nu}}(\alpha)=0$, so that $\partial_{\nu}\left(\alpha^{\prime}\right)$ is zero from the diagram above.

(2) $C_{\nu}$ is one of the lines $L_{x}, L_{y}$, or $L_{z}$. Note that modulo the equation of $C_{\nu}$, the element $d:=F(x, y, z)$ is a non-zero square, so that [P, Corollary 3.12] gives $\partial_{\nu}\left(\alpha^{\prime}\right)=0$. We deduce that, for any valuation $\nu$ of $K(Q)$ having a codimension-1 point in $\mathbb{P}_{\mathbb{C}}^{2}$ as center, the residue $\partial_{\nu}\left(\alpha^{\prime}\right)$ vanishes.

Codimension 2. Let $\mathfrak{p}_{\nu}$ be the center of $\nu$ on $\mathbb{P}^{2}$. We have an inclusion of local rings $\mathfrak{o}_{\mathbb{P}^{2}, \mathfrak{p}_{\nu}} \subset \mathfrak{o}_{\nu}$ inducing the inclusion of corresponding completions $\widehat{\mathcal{O}}_{\mathbb{P}^{2}, \mathfrak{p}_{\nu}} \subset \hat{\mathfrak{o}}_{\nu}$ with quotient fields $K_{\mathfrak{p}_{\nu}} \subset K(Q)_{\nu}$, respectively. We have three possibilities:

(1) If $\mathfrak{p}_{\nu} \notin L_{x} \cup L_{y} \cup L_{z}$, then $\alpha$ is a cup product of units in $\mathcal{O}_{\mathbb{P}^{2}, \mathfrak{p}_{\nu}}$, and hence units in $\mathfrak{o}_{v}$, so that $\partial_{\nu}\left(\alpha^{\prime}\right)=0$.

(2) If $\mathfrak{p}_{\nu}$ lies on one curve, e.g., $\mathfrak{p}_{\nu} \in L_{x} \backslash\left(\mathfrak{p}_{y} \sqcup \mathfrak{p}_{z}\right)$, where $\mathfrak{p}_{y}=(0,1,0)$ and $\mathfrak{p}_{z}=(0,0,1)$, then the image of $y$ in $\varkappa\left(\mathfrak{p}_{\nu}\right)$ is a non-zero complex number, and hence a square in $\widehat{\mathcal{O}}_{\mathbb{P}^{2}, \mathfrak{p}_{\nu}}$, and $y$ is also a square in $\hat{\mathfrak{o}}_{\nu}$. (We are using Hensel's lemma.) Thus, $\alpha^{\prime}=(x, y)=0$ in $H^{2}\left(K(Q)_{\nu}, \mathbb{Z} / 2 \mathbb{Z}\right)$, and $\partial_{\nu}\left(\alpha^{\prime}\right)=0$.

(3) If $\mathfrak{p}_{\nu}$ lies on two curves, e.g., $\mathfrak{p}_{\nu}=L_{x} \cap L_{y}$, then the image of $F(x, y, 1)$ in $\varkappa\left(\mathfrak{p}_{\nu}\right)$ is a non-zero complex number, and hence a square. By [P, Corollary 3.12], we have $\partial_{\nu}\left(\alpha^{\prime}\right)=0$.

\section{Singularities of the special fiber}

In this section we analyze the singularities of the fourfold introduced in Example 8 and studied in $\S 4$. Our main result is the following.

Proposition 11. The fourfold $X \subset \mathbb{P}^{2} \times \mathbb{P}^{3}$, with coordinates $(x, y, z)$ and $(s, t, u, v)$, respectively, given by

$$
y z s^{2}+x z t^{2}+x y u^{2}+F(x, y, z) v^{2}=0,
$$

with

$$
F(x, y, z)=x^{2}+y^{2}+z^{2}-2(x y+x z+y z),
$$

admits a universally $\mathrm{CH}_{0}$-trivial resolution of singularities.

We proceed as follows:

- identify the singular locus of $X$;

- construct a resolution of singularities $\beta: \widetilde{X} \rightarrow X$;

- verify universal $\mathrm{CH}_{0}$-triviality of $\beta$. 


\subsection{The singular locus}

Here we describe the singularities explicitly using affine charts on $\mathbb{P}^{2} \times \mathbb{P}^{3}$. The equations (5.1) and (5.2) are symmetric with respect to compatible permutations of $\{x, y, z\}$ and $\{s, t, u\}$. In addition, there is the symmetry

$$
(s, t, u, v) \longmapsto( \pm s, \pm t, \pm u, v)
$$

so altogether we have an action by the semidirect product $(\mathbb{Z} / 2 \mathbb{Z})^{3} \rtimes \mathfrak{S}_{3}$.

\section{Analysis in local charts}

Let $L_{x}, L_{y}, L_{z} \subset \mathbb{P}^{2}$ be the coordinate lines given by

$$
x=0, \quad y=0, \quad \text { and } \quad z=0,
$$

respectively, and

$$
\mathfrak{p}_{x}:=(1,0,0), \quad \mathfrak{p}_{y}:=(0,1,0), \quad \text { and } \quad \mathfrak{p}_{z}:=(0,0,1)
$$

be their intersections.

The quadrics in the family (5.1) drop rank over the coordinate lines $L_{x}, L_{y}$, and $L_{z}$, and over the conic $C \subset \mathbb{P}^{2}$, with equation

$$
F(x, y, z)=0
$$

where $F$ is defined in (5.2). This conic is tangent to the coordinate lines in the points

$$
\mathfrak{r}_{x}:=(0,1,1), \quad \mathfrak{r}_{y}:=(1,0,1), \quad \text { and } \quad \mathfrak{r}_{z}:=(1,1,0),
$$

respectively.

By symmetry, and since no singular point satisfies $s=t=u=0$, it suffices to consider just two affine charts:

Chart 1: $z=u=1$. Equation (5.1) takes the form

$$
y s^{2}+x t^{2}+x y+F(x, y, 1) v^{2}=0 .
$$

Derivatives with respect to $s, t$, and $v$ give

$$
y s=0, \quad x t=0, \quad \text { and } \quad v F(x, y, 1)=0 .
$$


Hence, from (5.3), $x y=0$. Derivatives with respect to $y$ and $x$ give

$$
s^{2}+x+(2 y-2 x-2) v^{2}=0 \quad \text { and } \quad t^{2}+y+(2 x-2 y-2) v^{2}=0 .
$$

Since $x y=0$, we have two cases, modulo symmetries:

Case 1: $y=0$;

Case 2: $x=0$ and $y \neq 0$.

We analyze each of these cases.

Case 1: $y=0$. Then $v F(x, y, 1)=0($ from $(5.4))$ implies

$$
v(x-1)^{2}=0 .
$$

So either $v=0$ or $x=1$. If $v=0$, from (5.5) we obtain $s^{2}+x=t=0$. Hence we obtain the following equations for the singular locus:

$$
y=v=t=s^{2}+x=0 .
$$

If $x=1$ then (5.4) implies $t=0$, and the remaining equation from (5.5) gives

$$
s^{2}+1-4 v^{2}=0
$$

Hence we obtain the following equations:

$$
x-1=y=t=s^{2}+1-4 v^{2}=0 .
$$

Case 2: $x=0$ and $y \neq 0$. From (5.4) the condition $y s=0$ implies $s=0$. There are two more cases: $v=0$ or $v \neq 0$. If $v=0$ the remaining equation (5.5) gives $t^{2}+y=0$. Hence we obtain equations for the singularity:

$$
x=v=s=t^{2}+y=0 .
$$

If $v \neq 0$, then $F(0, y, 1)=(y-1)^{2}=0$ from (5.4), hence $y=1$. The remaining equation from (5.5) gives

$$
t^{2}+y+(2 x-2 y-2) v^{2}=t^{2}+1-4 v^{2}=0 .
$$

So we obtain equations for the singular locus:

$$
x=y-1=s=t^{2}+1-4 v^{2}=0 .
$$

Chart 2: $z=s=1$. The equation of the quadric bundle is

$$
y+x t^{2}+x y u^{2}+F(x, y, 1) v^{2}=0 .
$$


As above, derivatives with respect to $t, u$, and $v$ give

$$
x t=0, \quad x y u=0, \quad \text { and } \quad v F(x, y, 1)=0 .
$$

Thus, from the equation, $y=0$. The conditions above and derivatives with respect to $y$ and $x$ yield

$$
x t=v(x-1)^{2}=1+x u^{2}+(-2 x-2) v^{2}=t^{2}+(2 x-2) v^{2}=0 .
$$

The second equation implies that either $x=1$ or $v=0$. If $x=1$, we obtain

$$
x-1=y=t=1+u^{2}-4 v^{2}=0 .
$$

If $v=0$, we obtain

$$
y=t=v=1+x u^{2}=0 .
$$

Collecting these computations, we obtain the following singularities:

(1) In Chart 1,

$$
\begin{array}{r}
y=v=t=s^{2}+x=0, \\
x-1=y=t=s^{2}+1-4 v^{2}=0, \\
x=v=s=t^{2}+y=0, \\
x=y-1=s=t^{2}+1-4 v^{2}=0 .
\end{array}
$$

(2) In Chart 2,

$$
\begin{array}{r}
x-1=y=t=1+u^{2}-4 v^{2}=0, \\
y=t=v=1+x u^{2}=0 .
\end{array}
$$

\section{Enumeration of strata}

Using the symmetries, we deduce that the singular locus of $X$ is a union of six conics. We distinguish between horizontal and vertical conics.

- The horizontal conics $C_{x}, C_{y}, C_{z} \subset X$ project onto the coordinate lines $L_{x}, L_{y}, L_{z} \subset$ $\mathbb{P}^{2}$. We express them using our standard coordinates on $\mathbb{P}^{2} \times \mathbb{P}^{3}$ :

$$
\begin{aligned}
& C_{y}=\left\{y=t=v=0 \text { and } z s^{2}+x u^{2}=0\right\}, \\
& C_{x}=\left\{x=s=v=0 \text { and } z t^{2}+y u^{2}=0\right\}, \\
& C_{z}=\left\{z=u=v=0 \text { and } x t^{2}+y s^{2}=0\right\} .
\end{aligned}
$$


These conics intersect transversally over $\mathfrak{p}_{z}, \mathfrak{p}_{x}, \mathfrak{p}_{y} \in \mathbb{P}^{2}$, respectively:

$$
C_{x} \cap C_{y}=\left\{\mathfrak{q}_{z}\right\}, \quad C_{y} \cap C_{z}=\left\{\mathfrak{q}_{x}\right\}, \quad \text { and } \quad C_{x} \cap C_{z}=\left\{\mathfrak{q}_{y}\right\}
$$

where

$$
\begin{aligned}
\mathfrak{q}_{z}:=(0,0,1) \times(0,0,1,0), & \pi\left(\mathfrak{q}_{z}\right)=\mathfrak{p}_{z} \\
\mathfrak{q}_{x}:=(1,0,0) \times(1,0,0,0), & \pi\left(\mathfrak{q}_{x}\right)=\mathfrak{p}_{x} \\
\mathfrak{q}_{y}:=(0,1,0) \times(0,1,0,0), & \pi\left(\mathfrak{q}_{y}\right)=\mathfrak{p}_{y}
\end{aligned}
$$

- The vertical conics $R_{y}, R_{x}, R_{z} \subset X$ project to the points $\mathfrak{r}_{y}, \mathfrak{r}_{x}, \mathfrak{r}_{z} \in \mathbb{P}^{2}$ :

$$
\begin{aligned}
& R_{y}=\left\{x-z=y=t=0 \text { and } s^{2}+u^{2}-4 v^{2}=0\right\} \\
& R_{x}=\left\{y-z=x=s=0 \text { and } t^{2}+u^{2}-4 v^{2}=0\right\} \\
& R_{z}=\left\{x-y=z=u=0 \text { and } s^{2}+t^{2}-4 v^{2}=0\right\} .
\end{aligned}
$$

These conics intersect the corresponding horizontal conics transversally in two points:

$$
R_{y} \cap C_{y}=\left\{\mathfrak{r}_{y+}, \mathfrak{r}_{y-}\right\}, \quad R_{x} \cap C_{x}=\left\{\mathfrak{r}_{x+}, \mathfrak{r}_{x-}\right\}, \quad \text { and } \quad R_{z} \cap C_{z}=\left\{\mathfrak{r}_{z+}, \mathfrak{r}_{z-}\right\},
$$

where

$$
\begin{aligned}
\mathfrak{r}_{y \pm}: & =(1,0,1) \times( \pm i, 0,1,0), \\
\mathfrak{r}_{x \pm}: & =(0,1,1) \times(0,1, \pm i, 0), \\
\mathfrak{r}_{z \pm}: & =(1,1,0) \times(1, \pm i, 0,0) .
\end{aligned}
$$

\section{Local étale description of the singularities}

The structural properties of the resolution become clearer after identifying étale normal forms for the singularities.

We now provide a local-étale description of the neighborhood of $\mathfrak{q}_{z}$. Equation (5.3) takes the form

$$
y s^{2}+x t^{2}+x y+F(x, y, 1) v^{2}=0 .
$$

At $\mathfrak{q}_{z}$ we have $F(x, y, 1) \neq 0$, so we can set

$$
v_{0}=v \sqrt{F(x, y, 1)}
$$


to obtain

$$
y s^{2}+x t^{2}+x y+v_{0}^{2}=0 .
$$

Set $x=m-n$ and $y=m+n$ to get

$$
(m+n) s^{2}+(m-n) t^{2}+m^{2}-n^{2}+v_{0}^{2}=0
$$

or

$$
m\left(s^{2}+t^{2}\right)+n\left(s^{2}-t^{2}\right)+m^{2}-n^{2}+v_{0}^{2}=0 .
$$

Then, let

$$
m=m_{0}-\frac{1}{2}\left(s^{2}+t^{2}\right) \quad \text { and } \quad n=n_{0}+\frac{1}{2}\left(s^{2}-t^{2}\right)
$$

to obtain

$$
m_{0}^{2}-n_{0}^{2}+v_{0}^{2}=\frac{1}{4}\left(\left(s^{2}+t^{2}\right)^{2}-\left(s^{2}-t^{2}\right)^{2}\right)=s^{2} t^{2} .
$$

We do a similar analysis in an étale-local neighborhood of either of the points $\mathfrak{r}_{y \pm}$. The singular strata for $C_{y}$ and $R_{y}$ are given in (5.6) and (5.7):

$$
\left\{y=t=v=s^{2}+x=0\right\} \quad \text { and } \quad\left\{y=t=x-1=s^{2}+1-4 v^{2}=0\right\} .
$$

We first introduce a new coordinate $w=x-1$. Thus, the singular stratum is the intersection of the monomial equations $y=t=v w=0$ with the hypersurface

$$
s^{2}+w+1-4 v^{2}
$$

We regard this as a local coordinate near $\mathfrak{r}_{y \pm}$. Equation (5.3) transforms to

$$
y s^{2}+w t^{2}+t^{2}+w y+y+v^{2}\left(-4 y+(w-y)^{2}\right)=0 .
$$

Regroup terms to obtain

$$
y\left(s^{2}+w+1-4 v^{2}\right)+t^{2}(1+w)=-v^{2}(w-y)^{2} .
$$

Now, note that $w \neq-1$, because $x \neq 0$ near $\mathfrak{r}_{y \pm}$. Let $t_{0}=t \sqrt{1+w}, s_{0}=s^{2}+w+1-4 v^{2}$, and $w_{0}=w-y$ to obtain

$$
y s_{0}+t_{0}^{2}=-v^{2} w_{0}^{2} .
$$

The normal forms (5.14) and (5.15) are both equivalent to

$$
a_{1}^{2}+a_{2}^{2}+a_{3}^{2}=\left(b_{1} b_{2}\right)^{2},
$$

with ordinary threefold double points along the lines

$$
\ell_{1}=\left\{a_{1}=a_{2}=a_{3}=b_{1}=0\right\} \quad \text { and } \quad \ell_{2}=\left\{a_{1}=a_{2}=a_{3}=b_{2}=0\right\} .
$$


A direct computation - which will be presented in $\S 5.2$ - shows that this is resolved by blowing up $\ell_{1}$ and $\ell_{2}$ in either order. The exceptional fibers over the generic points of $\ell_{1}$ and $\ell_{2}$ are smooth quadric surfaces, isomorphic to the Hirzebruch surface $\mathbb{F}_{0} \simeq \mathbb{P}^{1} \times \mathbb{P}^{1}$. Over the origin, we obtain a copy

$$
\mathbb{F}_{0} \cup_{\Sigma} \mathbb{F}_{2}
$$

where $\Sigma \simeq \mathbb{P}^{1}$ is the $(-2)$-curve on $\mathbb{F}_{2}$ and has self-intersection 2 on $\mathbb{F}_{0}$.

By symmetry, this analysis is valid at all nine special points

$$
\mathfrak{q}_{x}, \mathfrak{q}_{y}, \mathfrak{q}_{z}, \mathfrak{r}_{x \pm}, \mathfrak{r}_{y \pm}, \mathfrak{r}_{z \pm}
$$

where the components of the singular locus (the horizontal and vertical conics) intersect. This explains why we can blow these conics up in any order.

\subsection{Resolution of singularities}

\section{What we need to compute}

We propose blowing up as follows:

(1) blow up $C_{y}$;

(2) blow up the proper transform of $C_{x}$;

(3) blow up the proper transform of $C_{z}$;

(4) blow up the union of the proper transforms of $R_{x}, R_{y}$, and $R_{z}$, which are disjoint.

Taking into account the symmetry, after the first step we must understand:

- What are the singularities along the proper transform of $C_{x}$ ?

- What are the singularities along the proper transform of $R_{y}$ ?

Of course, answering the first questions clarifies the behavior along the proper transform of $C_{z}$. And $R_{x}$ and $R_{z}$ behave exactly the same as $R_{y}$.

Let $X_{1}$ denote the blow up of $C_{y}$ and $E_{1, y}$ the resulting exceptional divisor. We shall see that

- $E_{1, y}$ is smooth, except where it meets the proper transforms of $C_{x}, C_{z}$, and $R_{y}$;

- since $E_{1, y} \subset X_{1}$ is Cartier, $X_{1}$ is also smooth at any point of $E_{1, y}$, except where $E_{1, y}$ meets the proper transforms of $C_{x}, C_{z}$, and $R_{y}$;

- the fibers of $E_{1, y} \rightarrow C_{y}$ are smooth quadric surfaces away from $\mathfrak{q}_{x}, \mathfrak{q}_{z}$, and $\mathfrak{r}_{y \pm}$, over which the fibers are quadric cones.

Since the quadric bundle $E_{1, y} \rightarrow C_{y}$ admits sections, $E_{1, y}$ is rational over the function field of $C_{y}$ and all fibers of $E_{1, y} \rightarrow C_{y}$ are rational as well. 


\section{First blow up-local charts}

We describe the blow up of $C_{y}$ in charts. We start in Chart 1 , where $z=u=1$. Local equations for the center are given in (5.6), and we have a local chart for each defining equation.

- Chart associated with $y$. Equations for the blow up of the ambient space take the form

$$
v=y v_{1}, \quad t=y t_{1}, \quad \text { and } \quad s^{2}+x=y w_{1} .
$$

The equation of the proper transform of the quadric bundle is

$$
w_{1}+x t_{1}^{2}+F(x, y, 1) v_{1}^{2}=0 \quad \text { and } \quad s^{2}+x=y w_{1} .
$$

The exceptional divisor $E_{1, y}$ is given by $y=0$, i.e.,

$$
w_{1}+x t_{1}^{2}+(x-1)^{2} v_{1}^{2}=0 \text { and } \quad s^{2}+x=0 .
$$

The blow up is smooth at any point of the exceptional divisor in this chart, as the derivative of the first equation with respect to $w_{1}$ is 1 and the derivative of the second equation with respect to $w_{1}$ (resp. $x$ ) is 0 (resp. 1). (The proper transforms of $R_{y}$ and $C_{x}$ do not appear in this chart.) We analyze $E_{1, y} \rightarrow C_{y}$ : for any field $\varkappa / \mathbb{C}$ and $a \in \varkappa$, the fiber above $s=a, x=-a^{2}$, and $y=v=t=0$, is given by

$$
w_{1}-a^{2} t_{1}^{2}+\left(1+a^{2}\right)^{2} v_{1}^{2}=0
$$

which is smooth in this chart. Equation (5.16) makes clear that the exceptional divisor is rational and admits a section over the center.

- Chart associated with $s^{2}+x$. Equations for the blow up of the ambient space take the form

$$
y=\left(s^{2}+x\right) y_{1}, \quad v=\left(s^{2}+x\right) v_{1}, \quad \text { and } \quad t=\left(s^{2}+x\right) t_{1} .
$$

The proper transform of the quadric bundle has equation

$$
y_{1}+x t_{1}^{2}+F\left(x,\left(s^{2}+x\right) y_{1}, 1\right) v_{1}^{2}=0 .
$$

The exceptional divisor $E_{1, y}$ satisfies

$$
y_{1}+x t_{1}^{2}+(x-1)^{2} v_{1}^{2}=0 \quad \text { and } \quad s^{2}+x=0 .
$$


The blow up is smooth at any point of the exceptional divisor in this chart. (Again, the proper transforms of $R_{y}$ and $C_{x}$ do not appear in this chart.) The fiber above $s=a$, $x=-a^{2}$, and $y=v=t=0$ is given by

$$
y_{1}-a^{2} t_{1}^{2}+\left(1+a^{2}\right)^{2} v_{1}^{2}=0,
$$

which is smooth and rational in this chart.

- Chart associated with $t$. Equations for the blow up of the ambient space are

$$
y=t y_{1}, \quad v=t v_{1}, \quad \text { and } \quad s^{2}+x=t w_{1}
$$

and the proper transform of the quadric bundle satisfies

$$
y_{1} w_{1}+x+F\left(x, t y_{1}, 1\right) v_{1}^{2}=0 \quad \text { and } \quad s^{2}+x=t w_{1} .
$$

The exceptional divisor is given by $t=0$, i.e.

$$
y_{1} w_{1}+x+(x-1)^{2} v_{1}^{2}=0 \text { and } s^{2}+x=0 .
$$

The blow up is smooth along the exceptional divisor, except at the point

$$
t=v_{1}=y_{1}=s=w_{1}=x=0,
$$

which lies over the point $\mathfrak{q}_{z}$. Thus, the only singularity is along the proper transform of $C_{x}$. The fiber above $s=a, x=-a^{2}$, and $y=v=t=0$ is given by

$$
y_{1} w_{1}-a^{2}+\left(1+a^{2}\right)^{2} v^{2}=0
$$

which is smooth in this chart unless $a=0$.

- Chart associated with $v$. The equations are

$$
y=v y_{1}, \quad t=v t_{1}, \quad \text { and } \quad s^{2}+x=v w_{1}
$$

and

$$
y_{1} w_{1}+x t_{1}^{2}+F\left(x, v y_{1}, 1\right)=0 \quad \text { and } \quad s^{2}+x=v w_{1} .
$$

The exceptional divisor is given by $v=0$, i.e.

$$
y_{1} w_{1}+x t_{1}^{2}+(x-1)^{2}=0 \text { and } s^{2}+x=0 .
$$

The blow up is smooth at any point of the exceptional divisor except for

$$
y_{1}=v=w_{1}=t_{1}=0, \quad x=1, \quad \text { and } \quad s= \pm i .
$$

Thus, the only singularities are along the proper transform of $\mathfrak{r}_{y}$. The fiber above $s=a, x=-a^{2}$, and $y=v=t=0$ is given by

$$
y_{1} w_{1}-a^{2} t_{1}^{2}+\left(1+a^{2}\right)^{2}=0,
$$

which is smooth in this chart, unless $a= \pm i$. 
What is missed on restricting to Chart 1 ? For $C_{y}$, we omit only

$$
C_{y} \cap C_{z}=\left\{\mathfrak{q}_{x}\right\}
$$

with $\mathfrak{q}_{x}=(1,0,0) \times(1,0,0,0)$, but the symmetry exchanging $x$ and $z$ (and $s$ and $u$ ) takes this to $\mathfrak{q}_{z}$, which lies over Chart 1 . For $R_{y}$, we omit the locus

$$
x-z=y=t=u=s^{2}-4 v^{2}=0,
$$

which equals $(1,0,1) \times( \pm 2,0,0,1)$. However, the same symmetry takes these to $(1,0,1) \times$ $(0,0, \pm 2,1)$, which is over Chart 1 . Thus, modulo symmetries, computations over Chart 1 cover these points as well.

\section{Singularities above $\mathfrak{p}_{z}$}

Our goal is to show explicitly that the singularity of the blow up in the exceptional divisor $E_{1, y}$ over $(x, y, z)=(0,0,1)=\mathfrak{p}_{z}$ is resolved on blowing up the proper transform of $C_{x}$. It suffices to examine the chart associated with $t$, where we have equations

$$
y_{1} w_{1}+x+F\left(x, t y_{1}, 1\right) v_{1}^{2}=0 \quad \text { and } \quad s^{2}+x=t w_{1},
$$

i.e.,

$$
\left(y_{1}+t\right) w_{1}-s^{2}+F\left(-s^{2}+t w_{1}, t y_{1}, 1\right) v_{1}^{2}=0 \quad \text { and } \quad s^{2}+x=t w_{1},
$$

and the proper transform of $C_{x}$ satisfies

$$
y_{1}+t=0 \quad \text { and } \quad w_{1}=s=v_{1}=0 .
$$

If we compute the singular locus for the equation (5.20) above, at the points of the exceptional divisor $t=0$ and above $x=0$, we recover the equations for the proper transform of $C_{x}$ in this chart.

We analyze $X_{2}$, the blow up along the proper transform of $C_{x}$. In any chart above $y_{1}=t=0$ we have $F=1$ so étale locally we can introduce a new variable $v_{2}=v_{1} \sqrt{F}$ to obtain

$$
\left(y_{1}+t\right) w_{1}-s^{2}+v_{2}^{2}=0 .
$$

After the change of variables $y_{2}=y_{1}+t$,

$$
y_{2} w_{1}-s^{2}+v_{1}^{2}=0
$$

the singular locus is $y_{2}=s=w_{1}=v_{2}=0$. Here, $t$ is a free variable corresponding to an $\mathbb{A}^{1}$-factor. This is the product of an ordinary threefold double point with a curve, thus is resolved on blowing up the singular locus. Note the exceptional divisor is a smooth quadric surface bundle over the proper transform of $C_{x}$, over this chart. (There is a singular fiber over the point where it meets the proper transform of $C_{z}$.) 
Singularities above $\mathfrak{r}_{y}=(1,0,1) \in \mathbb{P}^{2}$

By the analysis above, we have only to consider the chart of the first blow up associated with $v$. Recall that it is obtained by setting

$$
y=v y_{1}, \quad t=v t_{1}, \quad \text { and } \quad s^{2}+x=v w_{1},
$$

with equation

$$
y_{1} w_{1}+x t_{1}^{2}+F\left(x, v y_{1}, 1\right)=0 .
$$

The exceptional divisor is given by $v=0$. The proper transform $R_{y}^{\prime}$ of the conic $R_{y}$ described by the equations

$$
x-1=y=t=0 \quad \text { and } \quad s^{2}+1-4 v^{2}=0
$$

is then

$$
x-1=y_{1}=t_{1}=0, \quad w_{1}-4 v=0, \quad \text { and } \quad s^{2}+1-4 v^{2}=0 .
$$

We obtain these equations by inverting the local equation for the exceptional divisor. Eliminating $x$ from the equation of $X_{1}$ yields an equation that can be put in the form

$$
y_{1}\left(w_{1}-4 v\right)+\left(-s^{2}+v w_{1}\right) t_{1}^{2}+\left(s^{2}-v w_{1}+v y_{1}+1\right)^{2}=0 .
$$

Writing $w_{2}=w_{1}-4 v$, we obtain

$$
y_{1} w_{2}+\left(-s^{2}+v w_{2}+4 v^{2}\right) t_{1}^{2}+\left(s^{2}-v w_{2}-4 v^{2}+v y_{1}+1\right)^{2}=0
$$

The curve $R_{y}^{\prime}$ may be expressed as a complete intersection

$$
y_{1}=w_{2}=t_{1}=0 \quad \text { and } \quad \sigma:=\left(s^{2}-4 v^{2}+1\right)+v\left(y_{1}-w_{2}\right)=0 .
$$

The coefficient

$$
c:=-s^{2}+v w_{2}+4 v^{2}
$$

is non-vanishing along $R_{y}^{\prime}$ in this chart so we may introduce an étale local coordinate $t_{2}=t_{1} \sqrt{c}$. Then, our equation takes the form

$$
y_{1} w_{2}+t_{2}^{2}+\sigma^{2}=0
$$

In other words, we have ordinary threefold double points along each point of $R_{y}^{\prime}$. Blowing up $R_{y}^{\prime}$ resolves the singularity, and the exceptional divisor over $R_{y}^{\prime}$ is fibered in smooth quadric surfaces. 


\section{3. $\mathrm{CH}_{0}$-triviality of the resolution}

Let $E_{1, y}$ denote the exceptional divisor after blowing up $C_{y}$. We have seen that the projection $E_{1, y} \rightarrow C_{y}$ is a quadric surface bundle. The fibers are smooth away from $\mathfrak{q}_{x}$, $\mathfrak{q}_{z}$, and $\mathfrak{r}_{y \pm}$; over these points the fibers are quadric cones.

Let $E_{1, x}$ denote the exceptional divisor after blowing up the proper transform $C_{x}^{\prime}$ of $C_{x}$. The fibration $E_{1, x} \rightarrow C_{x}^{\prime}$ is also a quadric surface bundle. The fibers are smooth away from $\mathfrak{q}_{y}$ and $\mathfrak{r}_{x \pm}$, where the fibers are quadric cones.

Let $E_{1, z}$ denote the exceptional divisor on blowing up the proper transform $C_{z}^{\prime}$ of $C_{z}$, after the first two blow ups. Again, $E_{1, z} \rightarrow C_{z}^{\prime}$ is a quadric surface bundle, smooth away from $\mathfrak{r}_{z \pm}$; the fibers over these points are quadric cones.

Finally, we blow up the proper transforms $R_{x}^{\prime}, R_{y}^{\prime}$, and $R_{z}^{\prime}$ of the disjoint vertical conics. The local computations above show that the resulting fourfold $\widetilde{X}$ is smooth and the exceptional divisors

$$
E_{2, x} \rightarrow R_{x}^{\prime}, \quad E_{2, y} \rightarrow R_{y}^{\prime}, \quad \text { and } \quad E_{2, z} \rightarrow R_{z}^{\prime}
$$

are smooth quadric surface bundles with sections.

To summarize, fibers of $\beta: \widetilde{X} \rightarrow X$ are one of the following:

- if $x$ is not contained in any of the conics, then $\beta^{-1}(x)$ is a point;

- if $x$ is contained in exactly one of the conics, then $\beta^{-1}(x)$ is a smooth quadric surface isomorphic to $\mathbb{F}_{0}$; when $x$ is a generic point of one of the conics, then $\beta^{-1}(x)$ is rational over the residue field of $x$;

- if $x$ is contained in two of the conics, then $\beta^{-1}(x)=\mathbb{F}_{0} \cup_{\Sigma} \mathbb{F}_{2}$, where $\mathbb{F}_{2}$ is the proper transform of a quadric cone appearing as a degenerate fiber, $\Sigma \subset \mathbb{F}_{2}$ is the (-2)-curve, and $\Sigma \subset \mathbb{F}_{0}$ has self-intersection 2 .

By Proposition 3 and Example 2, we conclude that $\beta$ is universally $\mathrm{CH}_{0}$-trivial.

\section{Analysis of Hodge classes}

Our approach follows [V3, §2]. As explained in Proposition 6, a quadric surface bundle over a rational surface $\pi: X \rightarrow S$ is rational, provided $X$ admits an integral class of type $(2,2)$ meeting the fibers of $\pi$ in odd degree. Here, we analyze how these classes occur.

We start by reviewing the Hodge-theoretic inputs. Let $\mathcal{Y} \rightarrow B$ be the family of all smooth hypersurfaces in $\mathbb{P}^{2} \times \mathbb{P}^{3}$ of bidegree $(2,2)$, i.e., $B$ is the complement of the discriminant in $\mathbb{P}\left(\Gamma\left(\mathcal{O}_{\mathbb{P}^{2} \times \mathbb{P}^{3}}(2,2)\right)\right)$. For each $b \in B$, let $Y_{b}$ denote the fiber over $b$. The Lefschetz hyperplane theorem gives Betti/Hodge numbers

- $b_{2 i+1}\left(Y_{b}\right)=0$

- $b_{2}\left(Y_{b}\right)=h^{1,1}\left(Y_{b}\right)=2$ and $b_{6}\left(Y_{b}\right)=h^{3,3}\left(Y_{b}\right)=2$. 
We compute $b_{4}\left(Y_{b}\right)$ by analyzing $Y_{b} \rightarrow \mathbb{P}^{2}$; its degeneracy divisor is an octic plane curve $D_{b}$, of genus 21 . Indeed, the fibers away from $D_{b}$ are smooth quadric surfaces, and the fibers over $D_{b}$ are quadric cones, so we have

$$
\chi\left(Y_{b}\right)=\chi\left(\mathbb{P}^{1} \times \mathbb{P}^{1}\right) \chi\left(\mathbb{P}^{2} \backslash D_{b}\right)+\chi(\text { quadric cone }) \chi\left(D_{b}\right)=4(3-(-40))+3(-40)=52 .
$$

It follows that $b_{4}\left(Y_{b}\right)=46$.

We extract the remaining Hodge numbers using techniques of Griffiths for hypersurfaces in projective space, extended to the toric case by Batyrev and Cox. Let $F$ be the defining equation of bidegree $(2,2)$ and consider the bigraded Jacobian ring:

$$
\operatorname{Jac}(F)=\mathbb{C}[x, y, z ; s, t, u, v] / \mathcal{I}(F)
$$

where $\mathcal{I}(F)$ is the ideal of partial derivatives of $F$. Note these derivatives satisfy the Euler relations

$$
x \frac{\partial F}{\partial x}+y \frac{\partial F}{\partial y}+z \frac{\partial F}{\partial z}=2 F=s \frac{\partial F}{\partial s}+t \frac{\partial F}{\partial t}+u \frac{\partial F}{\partial u}+v \frac{\partial F}{\partial v}
$$

Consider the vanishing cohomology

$$
H^{4}\left(Y_{b}\right)_{\mathrm{van}}:=H^{4}\left(Y_{b}\right) / H^{4}\left(\mathbb{P}^{2} \times \mathbb{P}^{3}\right),
$$

i.e., we quotient by $\left\langle h_{1}^{2}, h_{1} h_{2}, h_{2}^{2}\right\rangle$, where $h_{1}$ and $h_{2}$ are pull-backs of the hyperplane classes of $\mathbb{P}^{2}$ and $\mathbb{P}^{3}$, respectively. Then, we have [BC, Theorem 10.13]

- $H^{4,0}\left(Y_{b}\right)=H^{4,0}\left(Y_{b}\right)_{\mathrm{van}}=\operatorname{Jac}(F)_{(-1,-2)}=0$;

- $H^{3,1}\left(Y_{b}\right)=H^{3,1}\left(Y_{b}\right)_{\operatorname{van}} \simeq \operatorname{Jac}(F)_{(1,0)}=\mathbb{C}[x, y, z]_{1} \simeq \mathbb{C}^{3}$;

- $H^{2,2}\left(Y_{b}\right)_{\mathrm{van}} \simeq \operatorname{Jac}(F)_{(3,2)} \simeq \mathbb{C}^{37}$;

- $H^{1,3}\left(Y_{b}\right)=H_{1,3}\left(Y_{b}\right)_{\operatorname{van}} \simeq \operatorname{Jac}(F)_{(5,4)} \simeq \mathbb{C}^{3}$.

The first two dimension computations imply the others by the formula

$$
b_{4}\left(Y_{b}\right)=\sum_{p+q=4} h^{p, q}\left(Y_{b}\right)
$$

or one may compute the Hilbert function of an ideal generated by three forms of degree $(1,2)$ and four forms of degree $(2,1)$, subject to the relations $(6.1)$ but otherwise generic.

We recall the technique of Green [CHM, §5] and Voisin [V1, proof of §5.3.4], which applies as our variation of Hodge structures is effective of weight 2 after a suitable Tate twist. 
Proposition 12. Suppose that there exist $b_{0} \in B$ and $\gamma \in H^{2,2}\left(Y_{b_{0}}\right)_{\text {van }}$ such that the infinitesimal period map evaluated at $\gamma$,

$$
\bar{\nabla}(\gamma): T_{B, b_{0}} \longrightarrow H^{1,3}\left(Y_{b_{0}}\right),
$$

is surjective. Then, there exists a Zariski-dense set of $b \in B$ such that, for any simply connected Euclidean neighborhood $B^{\prime}$ of $b$, the image of the natural map (composition of inclusion with local trivialization)

$$
\tau_{b}: \mathcal{H}_{\mathbb{R}}^{2,2} \longrightarrow H^{4}\left(Y_{b}, \mathbb{R}\right)_{\mathrm{van}}
$$

contains an open subset $V_{b} \subset H^{4}\left(Y_{b}, \mathbb{R}\right)_{\text {van. }}$ Here, $\mathcal{H}_{\mathbb{R}}^{2,2}$ is a vector bundle over $B^{\prime}$ with fiber

$$
\mathcal{H}_{\mathbb{R}, u}^{2,2}=H^{4}\left(Y_{u}, \mathbb{R}\right)_{\operatorname{van}} \cap F^{2} H^{4}\left(Y_{u}, \mathbb{C}\right)_{\text {van }}
$$

over $u \in B^{\prime}$.

Note that the image is the set of real degree- 4 vanishing classes that are of type $(2,2)$ for some $b^{\prime} \in B^{\prime}$.

The infinitesimal condition is easy to check here. Since

$$
B \subset \mathbb{P}\left(\Gamma\left(\mathcal{O}_{\mathbb{P}^{2} \times \mathbb{P}^{3}}(2,2)\right)\right),
$$

we may identify

$$
T_{B, b_{0}}=\left(\mathbb{C}[x, y, z ; s, t, u, v] /\left\langle F_{0}\right\rangle\right)_{(2,2)},
$$

where $F_{0}$ is the defining equation of $Y_{b_{0}}$. The infinitesimal period map

$$
T_{B, b_{0}} \longrightarrow \operatorname{Hom}\left(H^{2,2}\left(Y_{b}\right), H^{1,3}\left(Y_{b}\right)\right)
$$

was interpreted by Carlson and Griffiths as a multiplication map

$$
\left(\mathbb{C}[x, y, z ; s, t, u, v] /\left\langle F_{0}\right\rangle\right)_{(2,2)} \times \operatorname{Jac}\left(F_{0}\right)_{(3,2)} \longrightarrow \operatorname{Jac}\left(F_{0}\right)_{(5,4)} .
$$

For fixed $\gamma \in \operatorname{Jac}\left(F_{0}\right)_{(3,2)}$, the differential in Voisin's hypothesis is computed by multiplying $\gamma$ with the elements of bidegree $(2,2)$ [V1, Theorem 6.13].

Example 13. Consider the hypersurface $Y_{b_{0}} \subset \mathbb{P}^{2} \times \mathbb{P}^{3}$ with equation

$$
\begin{aligned}
F_{0}=( & \left.u^{2}+u v+t s\right) x^{2}+\left(-t^{2}+u^{2}-v^{2}-s^{2}\right) x y+\left(t^{2}+u v+t s\right) y^{2} \\
& +\left(-t^{2}+u^{2}-v^{2}-s^{2}\right) x z+\left(t^{2}-16 t u-u^{2}+v^{2}+s^{2}\right) y z+\left(-3 u v-3 t s+s^{2}\right) z^{2} .
\end{aligned}
$$

We computed the Jacobian ring using Macaulay2 [GS]. In particular, we verified that

- $\operatorname{Jac}\left(F_{0}\right)_{\left(m_{1}, m_{2}\right)}=0$ for

$$
\left(m_{1}, m_{2}\right) \geqslant(13,2),(7,3),(3,5),
$$

so, in particular, $Y_{b_{0}}$ is smooth;

- the monomials $\left\{x z^{4} v^{4}, y z^{4} v^{4}, z^{5} v^{4}\right\}$ form a basis for $\operatorname{Jac}\left(F_{0}\right)_{(5,4)}$. 
Setting $\gamma=z^{3} v^{2}$, the multiples of $\gamma$ generate $\operatorname{Jac}\left(F_{0}\right)_{(5,4)}$. Hence, this example satisfies Voisin's hypothesis on the differential of the period map.

Proposition 14. Consider the Noether-Lefschetz loci

$$
\begin{aligned}
& \left\{b \in B: Y_{b} \text { admits an integral class of type }(2,2)\right. \\
& \text { meeting the fibers of } \left.Y_{b} \rightarrow \mathbb{P}^{2} \text { in odd degree }\right\} .
\end{aligned}
$$

These are dense in the Euclidean topology on B.

Proof. We retain the set-up of Proposition 12. The intersection of the NoetherLefschetz loci with $B^{\prime}$ may be expressed as

$$
\left\{u \in B^{\prime}: \mathcal{H}_{\mathbb{R}, u}^{2,2} \cap \tau_{b}^{-1} H^{4}\left(Y_{b}, \mathbb{Q}\right)_{\text {van }} \neq 0\right\}
$$

The density of the Noether-Lefschetz loci reflects the fact that

$$
H^{4}\left(Y_{b}, \mathbb{Q}\right)_{\text {van }} \subset H^{4}\left(Y_{b}, \mathbb{R}\right)_{\text {van }}
$$

is dense.

However, we are interested in vectors of $H^{4}\left(Y_{b}, \mathbb{Q}\right)_{\text {van }}$ that are rational multiples of those associated with odd degree multisections $M$ of $Y_{b} \rightarrow \mathbb{P}^{2}$. Such multisections exist because we can write a bidegree- $(2,2)$ hypersurface containing a constant section of $\mathbb{P}^{2} \times \mathbb{P}^{3} \rightarrow \mathbb{P}^{2}$. The parity condition corresponds to a congruence on the image of $M$ in $H^{4}\left(Y_{b}, \mathbb{Z}\right)_{\text {van. }}$ Indeed, write $\Lambda=H^{4}\left(Y_{b}, \mathbb{Z}\right)$ and consider the natural inclusions and homomorphisms

$$
\Lambda \supset\left\langle h_{1}^{2}, h_{1} h_{2}, h_{2}^{2}\right\rangle^{\perp \hookrightarrow} \Lambda /\left\langle h_{1}^{2}, h_{1} h_{2}, h_{2}^{2}\right\rangle=H^{4}\left(Y_{b}, \mathbb{Z}\right)_{\operatorname{van}}
$$

the cokernel of the middle arrow is the discriminant group of the lattice $\left\langle h_{1}^{2}, h_{1} h_{2}, h_{2}^{2}\right\rangle^{\perp}$, a finite abelian group. The class $M$ yields an element of this group and the parity condition translates into

$$
M \cdot h_{1}^{2} \equiv 1 \bmod 2
$$

Note that rational multiples of the elements satisfying this condition remain dense in $H^{4}\left(Y_{b}, \mathbb{R}\right)_{\text {van }}$, and so Proposition 12 gives the desired result.

The Noether-Lefschetz loci produced by this argument have codimension at most 3 in moduli; each is an algebraic subvariety of $B \subset \mathbb{P}\left(\Gamma\left(\mathcal{O}_{\mathbb{P}^{2} \times \mathbb{P}^{3}}(2,2)\right)\right) \simeq \mathbb{P}^{59}[\mathrm{CDK}]$. Any projective threefold in $\mathbb{P}^{59}$ will meet the closures of infinitely many of these loci. 


\section{Proof of Theorem 1}

We assemble the various ingredients developed above:

(1) Theorem 4 guarantees that a very general hypersurface of bidegree $(2,2)$ in $\mathbb{P}^{2} \times \mathbb{P}^{3}$ fails to be stably rational, provided we can find a special $X$ satisfying its hypotheses.

(2) The candidate example is introduced in Example 8.

(3) In $\S 4$, we show that $X$ has non-trivial unramified second cohomology. This verifies the first hypothesis of Theorem 4.

(4) In $\S 5$, we analyze the singularities of $X$, checking that it admits a resolution with universally $\mathrm{CH}_{0}$-trivial fibers.

(5) Proposition 6 gives a cohomological sufficient condition for rationality of $(2,2)$ hypersurfaces in $\mathbb{P}^{2} \times \mathbb{P}^{3}$; Proposition 14 shows that this condition is satisfied over a dense subset of the moduli space.

Consider a family $\phi: \mathcal{X} \rightarrow B$ of smooth $(2,2)$-hypersurfaces in $\mathbb{P}^{2} \times \mathbb{P}^{3}$ over a connected base $B$. If the base meets both the locus parameterizing non-stably rational varieties and the Noether-Lefschetz loci, then $\phi$ has both rational and irrational fibers.

Remark 15. Concrete examples of rational hypersurfaces $X_{\text {rat }} \subset \mathbb{P}^{2} \times \mathbb{P}^{3}$ of bidegree $(2,2)$ are easy to produce, e.g., those containing a constant section of the first projection. Any very general pencil containing $X_{\text {rat }}$ will have both rational and irrational fibers.

\section{References}

[A] Arason, J.K., Cohomologische Invarianten quadratischer Formen. J. Algebra, 36 (1975), 448-491.

[BC] Batyrev, V. V. \& Cox, D. A., On the Hodge structure of projective hypersurfaces in toric varieties. Duke Math. J., 75 (1994), 293-338.

[CDK] Cattani, E., Deligne, P. \& Kaplan, A., On the locus of Hodge classes. J. Amer. Math. Soc., 8 (1995), 483-506.

[CHM] Ciliberto, C., Harris, J. \& Miranda, R., General components of the NoetherLefschetz locus and their density in the space of all surfaces. Math. Ann., 282 (1988), 667-680.

[CT] Colliot-ThÉLÈne, J.-L., Birational invariants, purity and the Gersten conjecture, in K-Theory and Algebraic Geometry: Connections with Quadratic Forms and Division Algebras (Santa Barbara, CA, 1992), Proc. Sympos. Pure Math., 58, pp. 1-64. Amer. Math. Soc., Providence, RI, 1995.

[CTO] Colliot-Thélène, J.-L. \& Ojanguren, M., Variétés unirationnelles non rationnelles: au-delà de l'exemple d'Artin et Mumford. Invent. Math., 97 (1989), $141-158$.

[CTPa] Colliot-Thélène, J.-L. \& Pirutka, A., Hypersurfaces quartiques de dimension 3: non-rationalité stable. Ann. Sci. Éc. Norm. Supér., 49 (2016), 371-397. 
[CTPo] Colliot-Thélène, J.-L. \& Piryutko, E. V., Cyclic covers that are not stably rational. Izv. Ross. Akad. Nauk Ser. Mat., 80 (2016), 35-48 (Russian); English translation in Izv. Math., 80 (2016), 665-677.

[CTV] Colliot-ThÉlène, J.-L. \& Voisin, C., Cohomologie non ramifiée et conjecture de Hodge entière. Duke Math. J., 161 (2012), 735-801.

[dFF] De Fernex, T. \& Fusi, D., Rationality in families of threefolds. Rend. Circ. Mat. Palermo, 62 (2013), 127-135.

[GS] Grayson, D. R. \& Stillman, M. E., Macaulay2, a software system for research in algebraic geometry. Available at http://www.math.uiuc.edu/Macaulay2.

[H] Hassett, B., Some rational cubic fourfolds. J. Algebraic Geom., 8 (1999), 103-114.

[HKT] Hassett, B., Kresch, A. \& Tschinkel, Y., Stable rationality and conic bundles. Math. Ann., 365 (2016), 1201-1217.

[K] Kahn, B., Formes quadratiques sur un corps. Cours Spécialisés, 15. Société Mathématique de France, Paris, 2008.

[P] Pirutka, A., Varieties that are not stably rational, zero-cycles and unramified cohomology, in Algebraic Geometry (Salt Lake City, UT, 2015), Proc. Sympos. Pure Math., 97, pp. 459-484. Amer. Math. Soc., Providence, RI, 2018.

[S] Springer, T. A., Sur les formes quadratiques d'indice zéro. C. R. Acad. Sci. Paris, 234 (1952), 1517-1519.

[T1] Totaro, B., Hypersurfaces that are not stably rational. J. Amer. Math. Soc., 29 (2016), 883-891.

[T2] - Rationality does not specialise among terminal varieties. Math. Proc. Cambridge Philos. Soc., 161 (2016), 13-15.

[V1] Voisin, C., Hodge Theory and Complex Algebraic Geometry. II. Cambridge Studies in Advanced Mathematics, 77. Cambridge University Press, Cambridge, 2007.

[V2] - Unirational threefolds with no universal codimension 2 cycle. Invent. Math., 201 (2015), 207-237.

[V3] - (Stable) rationality is not deformation invariant. Preprint, 2015. arXiv: 1511.03591 [math.AG] .

Brendan Hassett

Department of Mathematics

Brown University

Box 1917

151 Thayer Street

Providence, RI 02912

U.S.A.

bhassett@math. brown.edu

YURI TSCHINKEL

Courant Institute

New York University

251 Mercer street

New York, NY 10012

U.S.A.

tschinkel@cims.nyu.edu

Received September 26, 2017

\author{
Alena Pirutka \\ Courant Institute \\ New York University \\ 251 Mercer street \\ New York, NY 10012 \\ U.S.A. \\ pirutka@cims.nyu.edu
}

